Western University

Scholarship@Western

Brain and Mind Institute Researchers'

Publications

Brain and Mind Institute

7-1-2015

\title{
Changes in visual and sensory-motor resting-state functional connectivity support motor learning by observing.
}

Heather R McGregor

The Brain and Mind Institute, Western University, London, Ontario, Canada; Department of Psychology, Western University, London, Ontario, Canada; Graduate Program in Neuroscience, Western University, London, Ontario, Canada

Paul L Gribble

The Brain and Mind Institute, Western University, London, Ontario, Canada; Department of Psychology, Western University, London, Ontario, Canada; Department of Physiology and Pharmacology, Schulich School of Medicine and Dentistry, Western University, London, Ontario, Canada, paul@gribblelab.org

Follow this and additional works at: https://ir.lib.uwo.ca/brainpub

Part of the Neurosciences Commons, and the Psychology Commons

Citation of this paper:

McGregor, Heather R and Gribble, Paul L, "Changes in visual and sensory-motor resting-state functional connectivity support motor learning by observing." (2015). Brain and Mind Institute Researchers' Publications. 197.

https://ir.lib.uwo.ca/brainpub/197 


\title{
Changes in visual and sensory-motor resting-state functional connectivity support motor learning by observing
}

\author{
Heather R. McGregor ${ }^{1,2,4}$ and Paul L. Gribble ${ }^{1,2,3}$ \\ ${ }^{1}$ The Brain and Mind Institute, Western University, London, Ontario, Canada; ${ }^{2}$ Department of Psychology, Western \\ University, London, Ontario, Canada; ${ }^{3}$ Department of Physiology and Pharmacology, Schulich School of Medicine and \\ Dentistry, Western University, London, Ontario, Canada; and ${ }^{4}$ Graduate Program in Neuroscience, Western University, \\ London, Ontario, Canada
}

Submitted 18 March 2015; accepted in final form 20 May 2015

\begin{abstract}
McGregor HR, Gribble PL. Changes in visual and sensory-motor resting-state functional connectivity support motor learning by observing. J Neurophysiol 114: 677-688, 2015. First published May 20, 2015; doi:10.1152/jn.00286.2015.-Motor learning occurs not only through direct first-hand experience but also through observation (Mattar AA, Gribble PL. Neuron 46: 153-160, 2005). When observing the actions of others, we activate many of the same brain regions involved in performing those actions ourselves (Malfait N, Valyear KF, Culham JC, Anton JL, Brown LE, Gribble PL. J Cogn Neurosci 22: 1493-1503, 2010). Links between neural systems for vision and action have been reported in neurophysiological (Strafella AP, Paus T. Neuroreport 11: 2289-2292, 2000; Watkins KE, Strafella AP, Paus T. Neuropsychologia 41: 989-994, 2003), brain imaging (Buccino G, Binkofski F, Fink GR, Fadiga L, Fogassi L, Gallese V, Seitz RJ, Zilles K, Rizzolatti G, Freund HJ. Eur J Neurosci 13: 400-404, 2001; Iacoboni M, Woods RP, Brass M, Bekkering H, Mazziotta JC, Rizzolatti G. Science 286: 2526-2528, 1999), and eye tracking (Flanagan JR, Johansson RS. Nature 424: 769-771, 2003) studies. Here we used a force field learning paradigm coupled with restingstate fMRI to investigate the brain areas involved in motor learning by observing. We examined changes in resting-state functional connectivity (FC) after an observational learning task and found a network consisting of V5/MT, cerebellum, and primary motor and somatosensory cortices in which changes in FC were correlated with the amount of motor learning achieved through observation, as assessed behaviorally after resting-state fMRI scans. The observed FC changes in this network are not due to visual attention to motion or observation of movement errors but rather are specifically linked to motor learning. These results support the idea that brain networks linking action observation and motor control also facilitate motor learning.
\end{abstract}

human; motor learning; observation; mirror neuron; resting-state fMRI

OUR CAPACITY FOR INTERACTING with the world depends on our facility for dexterous movement and the expansion of our motor repertoire through learning. How the brain achieves motor learning remains an important unresolved question and a problem of significant clinical importance, as many neurological conditions affect movement. Here we examine how action observation facilitates motor learning through functional plasticity in sensory and motor brain areas.

Motor learning is commonly studied with laboratory tasks in which subjects learn to adapt movements to counteract experimentally imposed changes in sensory feedback (Ghahramani et al. 1996) or mechanical perturbations (Shadmehr and Mussa-Ivaldi

Address for reprint requests and other correspondence: P. L. Gribble, The Brain and Mind Institute, Dept. of Psychology, 1151 Richmond St., NSC Bldg Rm. 120, London, ON, Canada N6A 5 B7 (e-mail: paul@gribblelab.org).
1994). Outside the laboratory, however, motor learning typically begins quite differently, often by observing a tutor. In human development, infant speech perception and speech motor learning are guided by visual and auditory observation of a caregiver's speech (Goldstein et al. 2003; Kuhl 2004; Kuhl et al. 1992). Observation also facilitates motor learning in adults. Mattar and Gribble (2005) showed that complex muscle force patterns can be learned by observing a tutor learning to reach in a novel force field (FF) environment. Subjects observed a tutor learning to perform straight reaches in a novel FF environment. Those subjects who were subsequently exposed to the same FF environment they had observed showed a performance benefit, executing straighter movements compared with nonobserving control subjects. Conversely, those subjects who were subsequently exposed to a different (opposite) FF environment than they had observed showed a significant disadvantage, performing more curved movements compared with the nonobserving control subjects (Mattar and Gribble 2005). These findings demonstrated that subjects had acquired representations of the observed dynamical environment.

The ability to learn about forces through observation is very interesting, since the observer cannot see the FF environment directly but only its consequences on the tutor's movements. Thus the observer only has access to visual information about the tutor's movement kinematics. To achieve motor learning, the brain must transform the visual information about the tutor's movements into the motor domain so as to allow the motor system to acquire a representation of the novel environment and the required changes to subsequent muscle force patterns.

A potential neural basis for the link between action and observation has emerged from the discovery of so-called mirror neurons in the premotor and parietal cortices of the macaque. These cells discharge while a monkey performs specific goal-directed actions and also while the animal observes another individual performing the same actions (di Pellegrino et al. 1992; Gallese et al. 1996; Rizzolatti et al. 1996). Neuroimaging studies support the idea that a similar system exists in the human brain (Buccino et al. 2001; Frey and Gerry 2006; Gallese et al. 2004). This putative human mirror neuron system is part of a broader action observation network (AON) including supplementary motor area (SMA), premotor, primary motor (M1) and primary somatosensory (S1) cortices, superior parietal lobule (SPL), and middle temporal visual area (V5/ MT) (Caspers et al. 2010). 
The functional role of the AON has been examined in prediction (Prinz 1997, 2006), action understanding (Rizzolatti et al. 1996; Rizzolatti and Fadiga 1998), and inferring action intentions (Gallese et al. 2004; Gallese and Goldman 1998), but surprisingly little work has addressed its potential role in motor learning (Buccino et al. 2004; Cross et al. 2009; Higuchi et al. 2012; Stefan et al. 2005).

Here we tested the hypothesis that brain areas linking observation and action are also involved in motor learning through observation. We used resting-state fMRI to examine functional connectivity (FC) before and after subjects observed a tutor learning to reach in a novel FF imposed by a robot arm. We describe a network involving visual area V5/MT, cerebellum, M1, and S1 in which changes in FC were correlated with subsequent behavioral measures of motor learning achieved through observation. Importantly, in a follow-up experiment we show that these FC changes are not explained by visual attention to motion or observation of movement errors but rather are specifically related to motor learning by observing. This network thus provides a neural basis by which visual information about the actions of others is propagated to sensory-motor circuits for learning.

\section{MATERIALS AND METHODS}

Subjects. Thirty healthy subjects participated in this study: 15 in the learning group in experiment 1 [ 6 men, 9 women; mean age $22.87 \pm$ $1.02(\mathrm{SE}) \mathrm{yr}$ ] and 15 in the control group in experiment 2 [6 men, 9 women; mean age $22.53 \pm 0.86(\mathrm{SE}) \mathrm{yr}$. All subjects were right handed and naive to FFs, had normal or corrected-to-normal vision, and reported no neurological or musculoskeletal disorders. Informed consent was obtained from all subjects before participation in procedures approved by the University of Western Ontario's Research Ethics Board.

Apparatus. Subjects sat at a custom tabletop and grasped the handle of a two-degree of freedom (DOF) robotic arm (IMT2, Interactive Motion Technologies) with their right hand (Fig. 1). An air sled was placed under the right arm to support the arm against gravity and

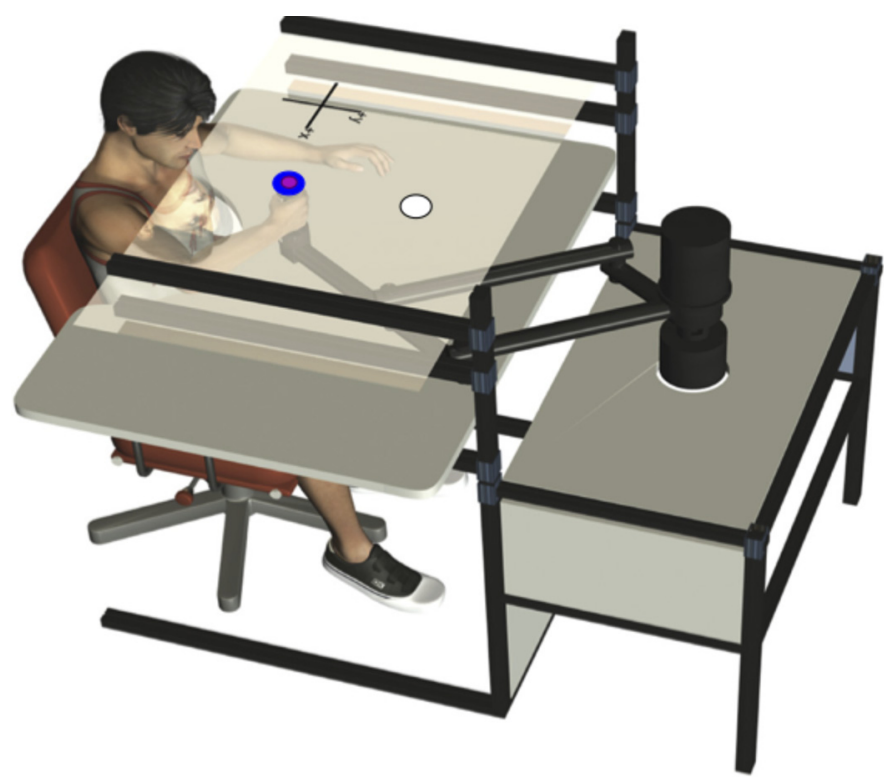

Fig. 1. Reaching task experimental setup. Subjects grasped the handle of a robotic arm with the right hand. A horizontal semisilvered mirror was mounted just above the robot handle. An LCD monitor (not shown) projected visual feedback onto the semisilvered mirror surface during the reaching task. reduce friction with the tabletop surface during the performance of reaching movements. The height of the chair was adjusted such that the subject's right arm (secured on the air sled) was abducted $90^{\circ}$ from the sagittal plane. The reaching task involved guiding the robot handle in a reaching movement to a visual target in the horizontal plane. A semisilvered mirror was mounted horizontally just above the robotic handle. An LCD monitor projected visual feedback onto the semisilvered mirror, including the start position (20-mm blue circle), the target (20-mm white circle), and a cursor representing hand position (12-mm pink circle) during the reaching task. Vision of the robotic arm and the subject's arm was occluded below the semisilvered mirror.

Subjects were instructed to perform reaching movements toward a single visual target while holding the handle of the robot arm. The target was located $15 \mathrm{~cm}$ in front of the start position along the sagittal plane. We instructed subjects to move directly to the target in a straight line without corrective movements. Upon the completion of each reach, the target changed color to provide visual feedback regarding movement duration. The target disappeared if the movement was within the desired time $(500 \pm 50 \mathrm{~ms})$, turned red if the movement was too fast, or turned green if the movement was too slow. These criteria were used to keep movement speed consistent and were not used to exclude trials. After feedback, the robot returned the subject's hand to the start position along a straight trajectory.

The robot was programmed to alter limb dynamics through the application of force on the subject's hand during movements. The robot applied a clockwise FF to deflect the hand rightward (right FF) or a counterclockwise FF to deflect the limb leftward (left FF). Velocity-dependent FFs were applied according to the following equation:

$$
\left[\begin{array}{l}
F_{x} \\
F_{y}
\end{array}\right]=\left[\begin{array}{cc}
0 & d k \\
-d k & 0
\end{array}\right]\left[\begin{array}{l}
v_{x} \\
v_{y}
\end{array}\right]
$$

where $x$ and $y$ are lateral and sagittal directions, $F_{x}$ and $F_{y}$ are the commanded forces, $v_{x}$ and $v_{y}$ are hand velocities, $k=14 \mathrm{~N} s / \mathrm{m}$, and $d=+1$ (right FF) or -1 (left FF).

Reaching video stimuli. Video stimuli showed a top-down view of a tutor reaching to a single target while holding the robotic arm with the right hand. Two video recordings were made, one in which a tutor learned to reach in a left FF and a second in which a different tutor reached in a random FF. Neither tutor had any previous experience reaching in a FF. A recording of the start position, target, and cursor was superimposed onto each video of the tutors' arm movements with Final Cut Pro 10 (Apple). A learning video was created that depicted the tutor performing 200 reaches as the robot applied a left FF (duration: $15 \mathrm{~min}$ ). This video was made with three 30 -s video clips from the left FF recording, each showing the typical progression from curved to straight movements during FF learning. The control video showed the tutor performing 200 reaches as the robot applied a FF that varied pseudorandomly from trial to trial between a left, right, or null FF (duration: $15 \mathrm{~min}$ ). The three 30-s clips used in this video thus showed movements that varied in their curvature but lacked the orderly progression from curved to straight movements depicted in the learning video. Maximum movement curvature was comparable between the learning and control videos. However, while the tutor in the learning video clips showed progressively decreasing movement curvature, the tutor in the control video clips showed consistently curved movements. A random force environment cannot be learned (Takahashi et al. 2001), and so the control video was used to test for changes in FC that may arise because of nonlearning factors such as visual attention to motion or observation of movement errors. Video clips were randomly ordered to create each video.

Experiment 1 design. Subjects $(n=15)$ participated in three sessions, each held on one of three consecutive days (Fig. $2 A$ ). On day $O$, subjects were familiarized with the robotic arm and performed 50 practice reaching trials in a null field, in which the robot did not apply 

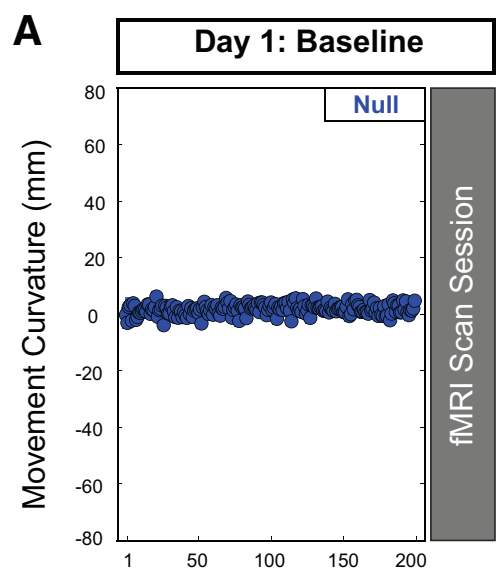
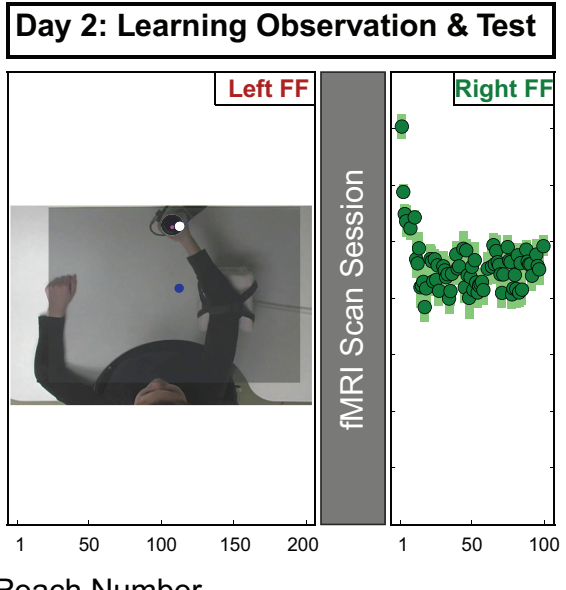

B

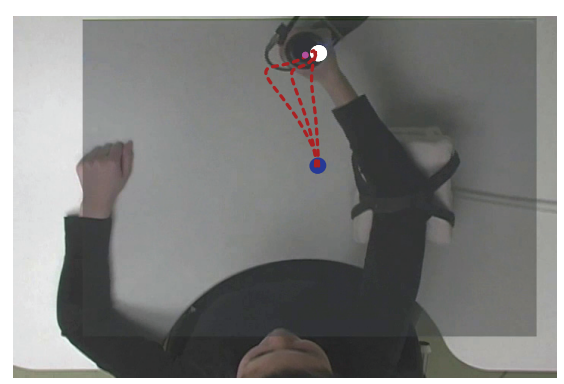

Reach Number

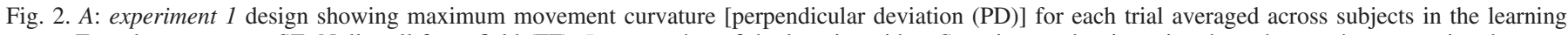

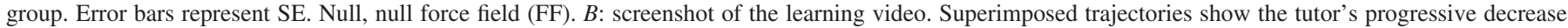
in movement curvature as the left FF is learned. Trajectories were not shown in the video.

force to the hand. Data from day 0 were not analyzed and have not been shown in Fig. $2 A$. The day 1 and day 2 sessions were held at the same time on each day. On day 1 , we acquired baseline measures. First, subjects performed 200 reaching trials in a null field to allow us to assess baseline movement curvature. Next, subjects walked to the imaging facility and underwent a baseline fMRI scan session. During the day 1 fMRI scan session (detailed description below), we measured baseline resting-state FC. The scan session began $\sim 20$ min after completion of the reaching task and lasted $60 \mathrm{~min}$. On day 2, subjects watched the video of the tutor learning to reach in a left FF (Fig. 2B) in the laboratory. Participants were seated in front of the robotic arm, and the LCD monitor projected the video onto the semisilvered mirror. While watching the video, subjects sat still with both arms resting on the tabletop (not holding the handle of the robot arm). We instructed subjects to count the total number of times the tutor in the video performed a reach at the desired speed (indicated by the target disappearing). Subjects reported the final tally to the experimenter at the end of the video. This was done to assess whether subjects were paying attention to the video. Tallies from subjects in the learning group are reported below in terms of accuracy. These data were not incorporated into behavioral or neuroimaging analyses. Next, subjects walked to the imaging facility and underwent a second fMRI scan session, which used the same protocol as on day 1. During the day 2 fMRI scan session, we again measured resting-state FC. This allowed us to assess how resting-state FC changed from baseline (day 1) to after observation (day 2). Finally, subjects walked back to the laboratory for a behavioral motor learning test. For the motor learning test, subjects performed 100 reaching movements while the robotic arm applied a rightward FF (test FF). This allowed us to assess the extent to which the learning and retention of the observed (left) FF interfered with performance in the opposite (right) test FF.

As has been used by Vahdat and colleagues (2011), Cothros and colleagues (2006), and Mattar and Gribble (2005), the direction of the test FF (right) was chosen to be opposite to the direction of the FF that acted on the tutor in the learning video (left). A hallmark of motor adaptation is the presence of aftereffects, that is, the continued use of a learned behavior when the environment is unexpectedly changed. For example, subjects who have learned to reach in a left FF have acquired a representation of the novel environment and the muscle force pattern required to counteract the applied leftward force by compensating rightward. Subjects continue to use the learned muscle force pattern even when the force environment is changed. For example, if the force environment was removed (null field), subjects' movements would initially be curved to the right (e.g., Shadmehr and Mussa-Ivaldi 1994). Aftereffects are particularly pronounced if the force environment changes such that it is the opposite of the learned (left) FF. When exposed to the right FF, the subject would compensate rightward (persistence of the learned muscle force pattern) in addition to being pushed rightward by the robotic arm. Thus those subjects who better learned the muscle force pattern required to perform straight reaches in the left FF would execute worse, more curved movements in the opposite (right) FF. In the present study, greater learning and retention of the observed left FF would bring about greater movement curvature when exposed to the right FF during the motor learning test (Brown et al. 2009; Cothros et al. 2006).

Experiment 2 design. We conducted a follow-up experiment to assess the extent to which the functional changes in the network identified in experiment 1 were specifically related to observational motor learning as opposed to other factors unrelated to learning, specifically visual attention to motion or observation of movement errors. A different group of naive subjects $(n=15)$ participated in experiment 2. As shown in Fig. $3 A$, the day 1 protocol was identical for both experiments 1 and 2. On day 2, control subjects watched the control video showing the tutor performing reaches in an unlearnable FF that randomly varied from trial to trial between a left, right, or null FF (Fig. 3B). Therefore, similar to the learning group, the control subjects observed movement errors and attended to visual motion while watching the video of the tutor. The critical difference was that the control subjects did not observe the tutor learning. As with the learning group, the control subjects observed the video of the tutor's movements in the laboratory while sitting in front of the robotic arm. Similarly, control subjects were instructed to count the number of times the tutor in the control video performed a reach at the correct speed (indicated by the white target disappearing) and to report the final tally to the experimenter. This was done as a check to verify that control subjects also paid attention to the video of the tutor's movements. Tallies from the control subjects are also reported below in terms of accuracy. These data were not incorporated into behavioral or neuroimaging analyses. As in experiment 1, control subjects underwent a second resting-state fMRI scan and a motor learning test (reaches in a right FF) after observation of the video of the tutor's movement (Fig. 3A). In summary, the control experiment used the same experimental design as experiment 1 , with the only exception that control subjects observed a tutor performing reaching movements with similar amounts of curvature as depicted in the learning video but not motor learning.

fMRI image acquisition. During each 1-h fMRI scan session, subjects underwent two 8-min resting-state scans under the instruction to remain awake with their eyes closed. The two resting-state runs were separated by a 5-min-long anatomical scan. During the anatom- 
A

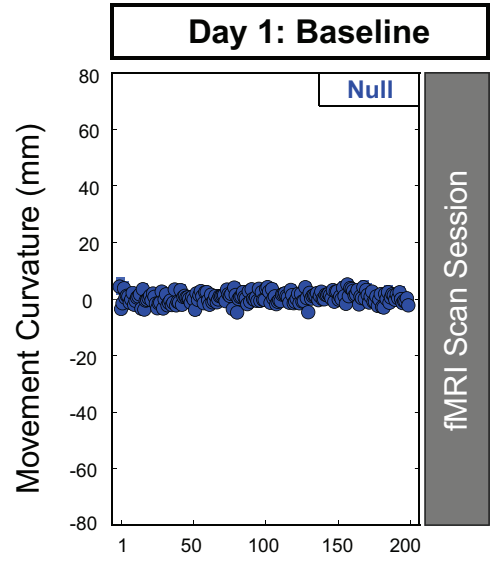

\section{Day 2: Control Observation \& Test}

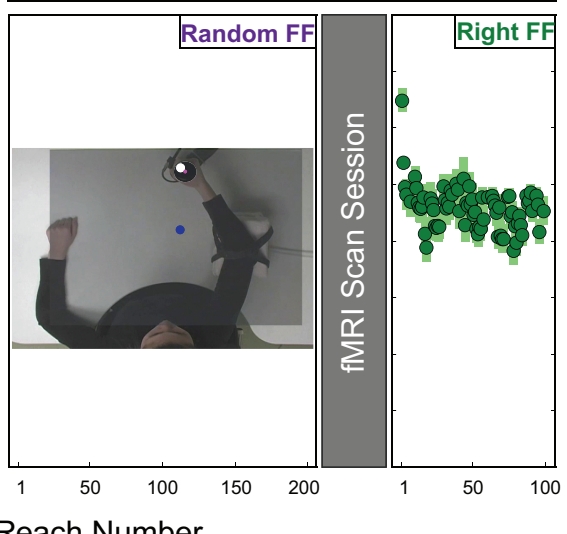

B

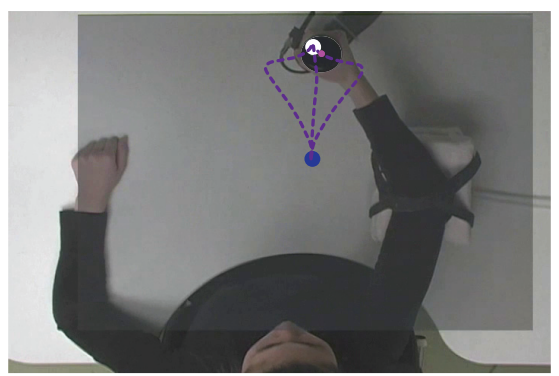

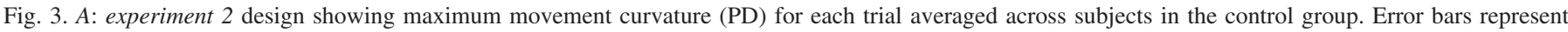

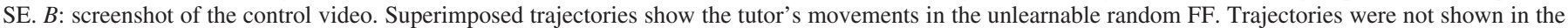
video.

ical scan, subjects were instructed to remain awake with their eyes open and fixate on a cross hair. Subjects then performed two 6-min functional localizer tasks. The localizer tasks were designed to allow us to determine the coordinates of 10 a priori-selected regions of interest (ROIs; see below) for use in the FC analyses described below.

The AON localizer task consisted of interleaved blocks of viewing intact and scrambled video clips of a tutor performing reaches while holding the robotic arm (ten 36-s blocks). Intact video clips showed a top-down view of a tutor performing straight reaching movements in a null FF. For the baseline condition the video clips were scrambled, with only the start position and target remaining in their original locations. This approach preserved the low-level motion-related features of the visual image such as movement direction and velocity while disrupting the details of the movement such as shoulder and elbow joint rotations and hand path curvature (Malfait et al. 2010). During observation of the AON localizer video, all subjects were instructed to count the total number of times the target disappeared and report it to the experimenter at the end of the video. This was done as a check to make sure each subject paid attention to the AON localizer video. Subjects' reported tallies were not incorporated into the behavioral or neuroimaging analyses.

The motor localizer task consisted of interleaved blocks of arm movement and rest (ten 36-s blocks). During movement blocks, subjects slowly moved their right forearm along the frontal plane in a cyclic manner $\left(90^{\circ}\right.$ elbow flexion). Movements were paced at a frequency of $0.1 \mathrm{~Hz}$ with color-coded visual cues.

Neuroimaging data were acquired with a 3-T Siemens Magnetom Tim Trio imaging system with a 32-channel head coil. Whole-brain functional data were acquired with a T2*-weighted EPI sequence $\left(\mathrm{TR}=3,000 \mathrm{~ms}, \mathrm{TE}=30 \mathrm{~ms}\right.$, flip angle $=90^{\circ}, 3-\mathrm{mm}$ isotropic voxels, $80 \times 80 \times 50$ matrix, iPAT acceleration factor $=2)$. The T1-weighted anatomical images were collected with a MPRAGE sequence $\left(\mathrm{TR}=2,300 \mathrm{~ms}, \mathrm{TE}=2.98 \mathrm{~ms}\right.$, flip angle $=9^{\circ}, 1-\mathrm{mm}$ isotropic voxels, $192 \times 240 \times 256$ matrix). For each scanning session a field map was acquired with a gradient echo sequence $(\mathrm{TR}=531$ $\mathrm{ms}$, TE $=4.92 \mathrm{~ms} / 7.38 \mathrm{~ms}$, flip angle $=60^{\circ}, 3-\mathrm{mm}$ isotropic voxels, $80 \times 80 \times 50$ matrix) .

Behavioral data analysis. The robot handle position, velocity, and applied force were sampled at $600 \mathrm{~Hz}$. The position data were low-pass filtered at $40 \mathrm{~Hz}$. The start and end of each trial were defined as the position at which the hand's velocity increased above and decreased below $5 \%$ of the peak velocity, respectively. Movement curvature was calculated for each trial as the maximum perpendicular deviation of the hand $(\mathrm{PD})$ relative to a straight line connecting the start position and reaching target (Mattar and Gribble 2005). Motor learning was assessed after the day 2 fMRI scan session, $\sim 80 \mathrm{~min}$ after subjects watched the reaching video. A motor learning score was calculated for each subject as the mean PD of the first 3 trials in the test FF relative to the mean PD of the last 50 trials in the null FF. In this way, we were able to quantify the extent to which the learning and retention of the observed FF interfered with each subject's subsequent performance in the (right) test FF. We also assessed movement curvature throughout the entire motor learning test. Individual PD scores were averaged over 10-trial blocks, and group means were compared with analysis of variance and Bonferroni-corrected post hoc tests.

fMRI image preprocessing. Neuroimaging data analyses were performed with FSL version 5.0.4 (FMRIB's Software Library, www. fmrib.ox.ac.uk/fsl). Image preprocessing consisted of the removal of the first 2 volumes in each functional run, slice-timing correction, motion correction, nonbrain tissue removal, spatial smoothing using a 6-mm FWHM Gaussian kernel, and high-pass temporal filtering (100 s). Field map distortion correction and linear coregistration of functional and anatomical images were performed with boundary-based registration (BBR) in FLIRT. Images were transformed into standard space (MNI's 152-brain T1 template, 2-mm isotropic voxel size) with a 12 -DOF linear registration.

fMRI regions of interest. We selected 10 a priori ROIs involved in action observation and/or motor learning (see Table 1). These regions included left SMA, dorsal premotor cortex (PMd), ventral premotor cortex (PMv), M1, S1, V5/MT, SPL, inferior parietal lobule (IPL), putamen (BG), and right cerebellum (CB). ROI coordinates were determined with the data acquired during the independent functional localizer tasks. While the localizer tasks were performed after the resting-state scans on both days, the seed region coordinates were defined with only the data collected from the localizer tasks performed during the day 1 (baseline) scan session. This was done to ensure that the data used to define seed regions were based on scans following identical experiences of the two groups. If we had also included day 2 scans to define seed regions, the BOLD response evoked by the localizer tasks might have differed across groups due to each group having observed a different video at the beginning of day 2 (learning vs. control).

The task-induced response for each localizer was assessed with a per-subject GLM. All 30 subjects were then included in a mixedeffects analysis of the localizer tasks (cluster threshold of $Z>2.3$, $P<0.05$ ). The 10 brain regions listed above were identified in the resulting $Z$ map with the Jülich histological (cyto- and myelo-archi- 
Table 1. Seed regions and coordinates used for resting-state functional connectivity analyses

\begin{tabular}{lccrc}
\hline \hline & \multicolumn{3}{c}{ MNI Coordinates } \\
\cline { 2 - 4 } ROI & $x$ & $y$ & $z$ & $Z$ \\
\hline SMA & -4 & -10 & 56 & 5.93 \\
PMd & -24 & -22 & 66 & 6.02 \\
PMv & -42 & -6 & 56 & 5.16 \\
M1 & -26 & -30 & 64 & 6.41 \\
S1 & -30 & -36 & 62 & 6.32 \\
V5/MT & -42 & -76 & 2 & 5.70 \\
SPL & -22 & -48 & 68 & 5.87 \\
IPL & -60 & -44 & 22 & 4.01 \\
BG & -28 & -14 & 8 & 4.52 \\
CB & 26 & -44 & -26 & 5.22 \\
\hline
\end{tabular}

ROI, region of interest; SMA, supplementary motor area; PMd, dorsal premotor cortex; PMv, ventral premotor cortex; M1, primary motor cortex; S1, primary somatosensory cortex; V5/MT, middle temporal visual area; SPL, superior parietal lobule; IPL, inferior parietal lobule; BG, putamen; CB, cerebellum.

tectonic) atlas (Eickhoff et al. 2005), the Harvard-Oxford subcortical structural atlas (Desikan et al. 2006), or the probabilistic cerebellar atlas (Diedrichsen et al. 2009). Within each of the 10 brain regions, the ROI coordinate was chosen as the peak voxel (Table 1). ROIs included all voxels within a 6-mm-radius sphere centered on the activation peaks. The same standard (MNI) space seed coordinates were used for both groups.

Functional connectivity analysis. The FC analysis was performed on both resting-state runs acquired on day 1 and both resting-state runs acquired on day 2. The ultimate goal of the analysis was to estimate how FC on day 2, after observation of learning, differs from baseline FC on day 1. Each preprocessed resting-state run was band-pass filtered, preserving frequencies between 0.01 and $0.1 \mathrm{~Hz}$, within which resting-state activity is observed (Biswal et al. 1995; Damoiseaux et al. 2006). Intensity normalization was performed by scaling the mean value of each run to 10,000 to factor out global intensity differences between resting-state runs. Seed-based correlation analyses were then carried out for each subject to assess FC between each seed ROI and the rest of the brain. The mean time series from each ROI was used as a predictor in a whole-brain regression analysis implemented in FILM (FMRIB's Improved General Linear Model). The following signals were included in the model as nuisance regressors: the temporal derivative of the ROI signal, six rigid body motion parameters obtained from motion correction, mean global signal, mean white matter signal, and mean CSF signal. All signals were band-pass filtered $(0.01-0.1 \mathrm{~Hz})$ prior to nuisance regression.

The resulting contrast images were entered into a mixed-effects model (FLAME) for each group. In this analysis, the binary GLM predictor modeling the change in $\mathrm{FC}$ from day 1 to day 2 was weighted by each subject's respective motor learning score (Vahdat et al. 2011). In this way, the resulting change in FC can be linked to the behavioral measure of motor learning. The predictor of interest reflecting subject behavior was orthogonalized with respect to regressors modeling each subject's overall mean. Corrections for multiple comparisons were carried out at the cluster level $(Z>2.6$, cluster significance: $P<0.05$, corrected for familywise error with Gaussian random field theory). We further applied a Bonferroni correction for the number of ROIs examined; thus reported clusters are those that survived a size threshold of $P<0.005$ (i.e., $P<0.05 / 10$ ROIs). These analyses yielded $Z$ score maps reflecting networks whose changes in FC from day 1 to day 2 were related to our behavioral measure of motor learning achieved. FC was defined as the temporal correlation (Fisher Z-transformed correlation coefficients) between the seed region time course and the average time course of target clusters.

\section{RESULTS}

Experiment 1: motor learning by observing. Observing the tutor learning a left FF affected subjects' subsequent motor behavior in the final test FF. Subjects who observed left FF learning performed significantly worse (i.e., more curved) movements when they later encountered the test (right) FF compared with control subjects who observed similar curved movements in an unlearnable random FF (experiment 2). The learning group's poorer performance in the test FF is indicative of proactive interference due to learning of the observed left FF (Brown et al. 2009; Cothros et al. 2006). Figure $4 A$ shows typical hand trajectories corresponding to the first movement in the test FF for representative subjects in each group. The behavioral difference between groups is indicated by reliably higher motor learning scores for the learning group [Fig. 4B; $t(28)=2.58, P<0.01]$. Figure $4 C$ shows mean movement curvature (PD) over the entire motor learning test, averaged over 10 trial blocks. Movements in the test FF were initially highly curved to the right but subsequently decreased in curvature over the course of trial blocks. While both groups reduced trajectory curvature in the test FF as a function of trial block, the pattern of the decrease depended on the FF that was previously observed $[F(9,252)=2.39, P<0.05$, GreenhouseGeisser corrected]. This behavioral difference was seen in the first block of the test FF movements, during which subjects who had observed left FF learning exhibited $40 \%$ greater trajectory curvature than control subjects $[t(28)=2.99, P<$ 0.03]. This result is consistent with previous observational

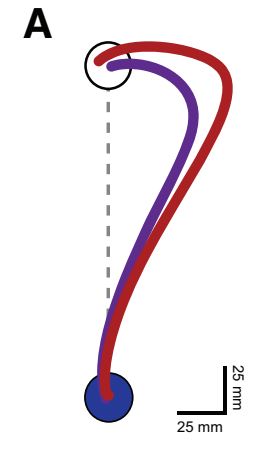

C

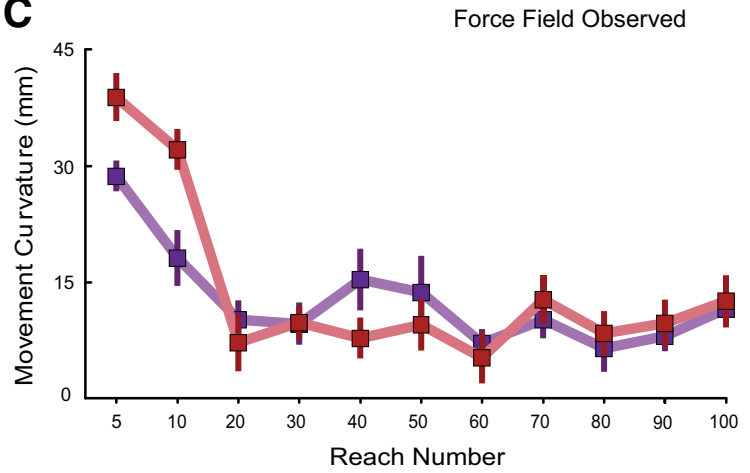

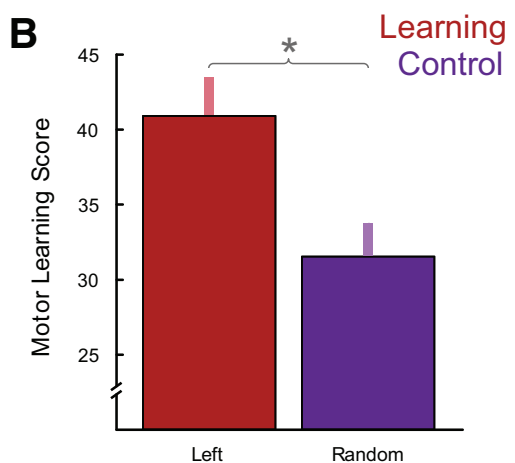

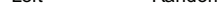

Force Field Observed
Fig. 4. Motor learning test performance. A: typical hand trajectories corresponding to the 1 st movement in the test (right) FF after having observed left FF learning (red) or movements in an unlearnable random FF (control, purple). $B$ : motor learning scores as a function of FF observed. $* P<0.01$. Error bars represent SE. $C$ : maximum movement curvature (PD) throughout the entire motor learning test. The first 10 movements are shown as averages of 5-trial blocks. Subsequent movements are shown as averages of 10-trial blocks. Error bars represent SE. 
learning studies (Bernardi et al. 2013; Brown et al. 2009; Mattar and Gribble 2005; Williams and Gribble 2012).

To investigate brain areas involved in motor learning by observing, we examined postobservation changes in FC (day 2 vs. day 1) involving our functionally defined ROIs (Table 1) that were related to subjects' respective motor learning scores (as assessed after the final resting-state scan on day 2). In this way, we sought to identify functional networks associated with the amount of motor learning achieved through observation.

These analyses yielded statistically reliable results only for our ROI in left V5/MT. Left V5/MT, an area involved in visual motion perception (Watson et al. 1993; Zeki et al. 1991), was the most highly activated region during our AON localizer task. Figure 5 shows target clusters that exhibited FC changes with left V5/MT that were related to motor learning scores. Figure 5, top, shows that left V5/MT exhibited decreased FC with a cluster in left cerebellar cortex (lobule VI and Crus I) after observation of left FF learning; as shown on the bar graph, the negative correlation between V5/MT and cerebellum on day 1 approached zero on day 2 after observation of FF learning. In Fig. 5, top right, we have presented the relationship between behavioral motor learning scores, used as regressors of interest in our FC analysis, and the changes in FC to further illustrate how subjects with higher motor learning scores exhibited greater decreases in FC between V5/MT and cerebellum. The nonindependence of this particular correlation may inflate the effect size; thus we have presented this analysis as a sanity check for illustrative purposes, not as the basis for inference (Poldrack and Mumford 2009). It is worth noting that while the FC between V5/MT and cerebellum decreased from day 1 to day 2, the scatterplot shows a positive correlation. This is due to the subtraction of negative (day 1) FC values from near-zero (day 2) FC values, yielding positive FC changes (day 1 and day 2 FC values are shown in Fig. 6). Figure 5, bottom, shows that left V5/MT also exhibited decreased FC with a cluster spanning left M1 and left S1; the bar graph shows that the positive correlation between V5/MT, M1 and S1 on day 1 approached zero on day 2 after observation. The scatterplot presents the relationship between behavioral motor learning scores and decreases in FC between V5/MT, M1 and S1, again illustrating that those subjects who achieved greater motor learning through observation exhibited greater FC decreases. Subjectwise FC values on day 1 (baseline) and day 2 (after observation) are shown in Fig. 6. Anatomical labels and local $Z$ value maxima for each cluster are reported in Table 2.

Experiment 2: control observation. To examine the extent to which the changes in the identified functional network are specifically related to motor learning by observing, we conducted a follow-up experiment in which subjects observed a tutor reaching in an unlearnable random FF.

During the motor learning test, control subjects' movements were significantly less curved upon initial exposure to the (right) test FF compared with those subjects who observed FF learning (Fig. 4), indicating less learning and retention of the

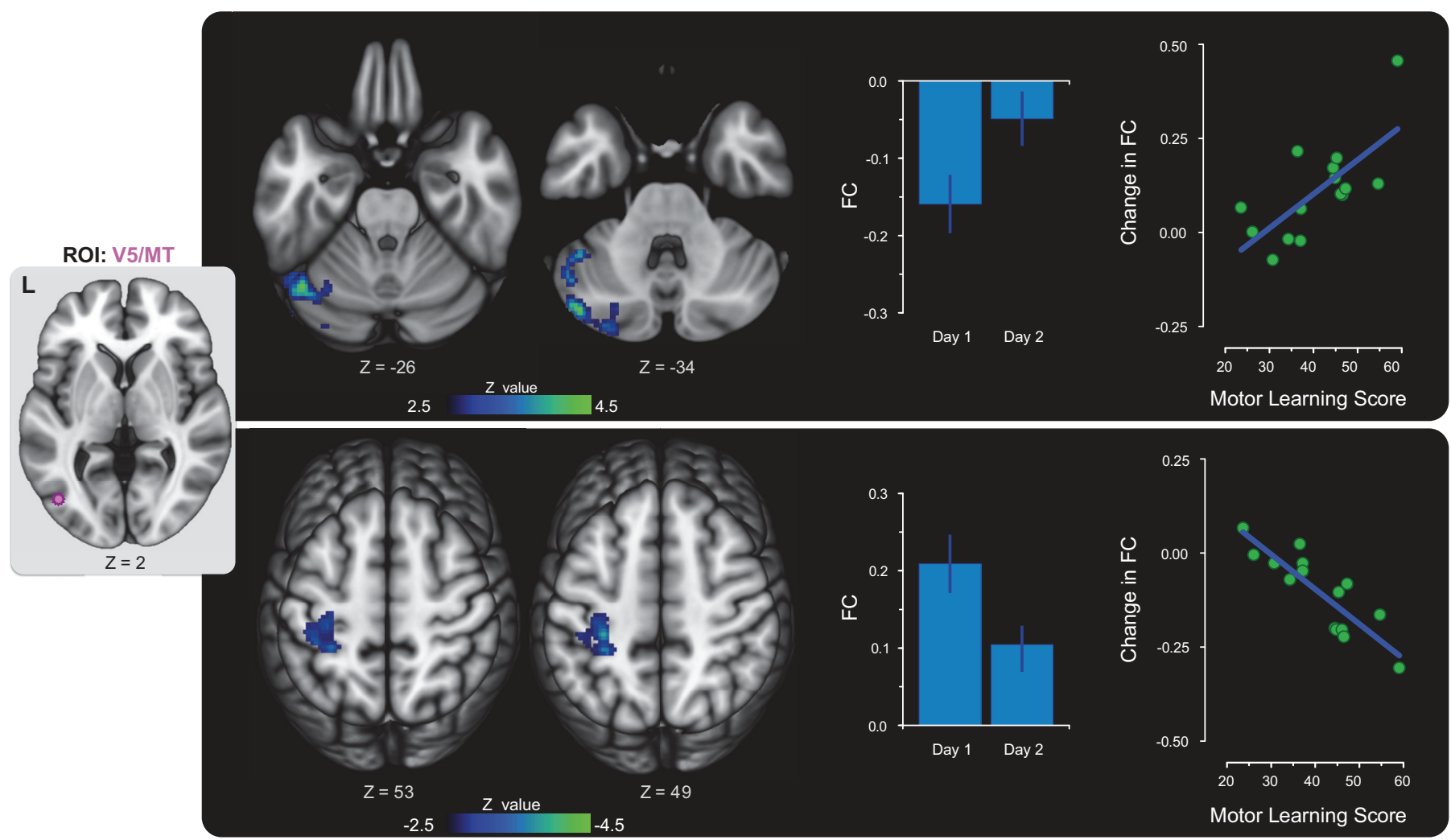

Fig. 5. Changes in resting-state functional connectivity (FC) from day 1 (baseline) to day 2 (after observation) related to motor learning scores. In the learning group, the region of interest (ROI) in V5/MT (inset at left) exhibited decreased FC with the cerebellum (top) and also primary motor (M1) and primary somatosensory (S1) cortices (bottom; $Z>2.6$, corrected for familywise error with Gaussian random field theory, $P<0.005$ ). Bar graphs show that FC between left V5/MT and each cluster decreased from day 1 to day 2 such that FC approached zero after observation of left FF learning. Scatterplots further illustrate that the observed functional changes are indeed related to our behavioral measure of motor learning, as assessed during the motor learning test after the day 2 fMRI scan. 

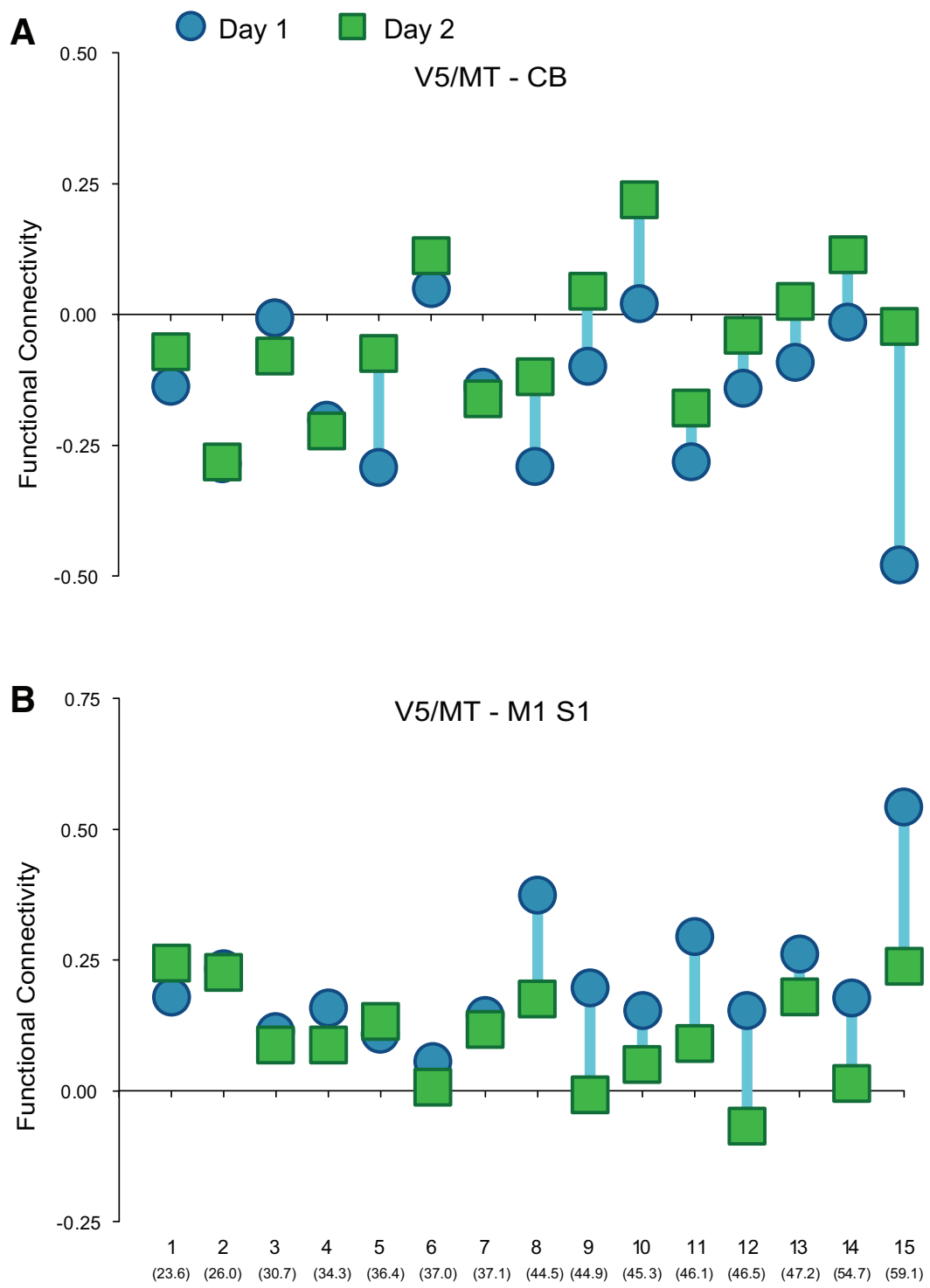

Subject

(Motor Learning Score)
Fig. 6. Subjectwise FC values on day 1 (baseline) and day 2 (after observation) for the learning group. Subjects are rank ordered along the $x$-axis according to increasing motor learning scores as indicated in parentheses. A: FC between V5/MT and cerebellum (CB) decreased from day 1 to day 2 such that subjects with higher motor learning scores showed greater decreases in FC (i.e., correlations approach 0). B: FC between V5/MT and M1 and S1 also decreased from day 1 to day 2 such that those subjects with higher motor learning scores exhibited greater decreases in FC (i.e., correlations approach 0). observed random FF. Similarly, their motor learning scores were reliably lower than subjects who observed left FF learning.

The FC analyses described above were also carried out for the control group. The same parameters were used for these analyses, including the same ROI coordinates. FC analyses for the control group yielded no statistically reliable clusters for the V5/MT seed region. To further explore these data, we repeated the group-level analysis using lower $Z$ thresholds. No significant target clusters appeared for the V5/MT ROI until the threshold was lowered to $Z>2.2$. Even at lower threshold levels, the clusters bear no resemblance to the learning group result (Fig. 7). In sum, this indicates that the FC changes within the functional network observed in the learning group are not

Table 2. Clusters in which changes in FC were correlated with motor learning scores

\begin{tabular}{|c|c|c|c|c|c|c|c|c|}
\hline \multirow[b]{2}{*}{ ROI } & \multirow[b]{2}{*}{ Cluster $P$ Value } & \multirow{2}{*}{$\begin{array}{c}\text { Local Maxima } \\
\text { Z Scores }\end{array}$} & \multicolumn{3}{|c|}{ MNI Coordinates } & \multirow{2}{*}{$\begin{array}{l}\text { Day } 1 Z \\
\text { Score }\end{array}$} & \multirow{2}{*}{$\begin{array}{l}\text { Day } 2 Z \\
\text { Score }\end{array}$} & \multirow[b]{2}{*}{ Anatomical Label } \\
\hline & & & $x$ & $y$ & $z$ & & & \\
\hline \multirow[t]{5}{*}{ Left V5/MT } & 0.00122 & 4.89 & -44 & -76 & -32 & -4.21 & -2.25 & Left cerebellum (Crus I) \\
\hline & & 4.27 & -38 & -66 & -22 & 0.58 & 1.90 & Left cerebellum (lobule VI) \\
\hline & 0.00406 & 4.00 & -30 & -32 & 46 & 4.23 & 1.82 & Left S1 (BA3a) \\
\hline & & 3.60 & -30 & -40 & 50 & 4.49 & 2.45 & Left S1 (BA2) \\
\hline & & 3.37 & -32 & -28 & 50 & 3.63 & 1.37 & Left M1 (BA4p) \\
\hline
\end{tabular}

Functional connectivity (FC) data corresponding to the cerebellar cluster are shown in the top rows, and data corresponding to the sensory-motor cluster are shown in the bottom rows. 


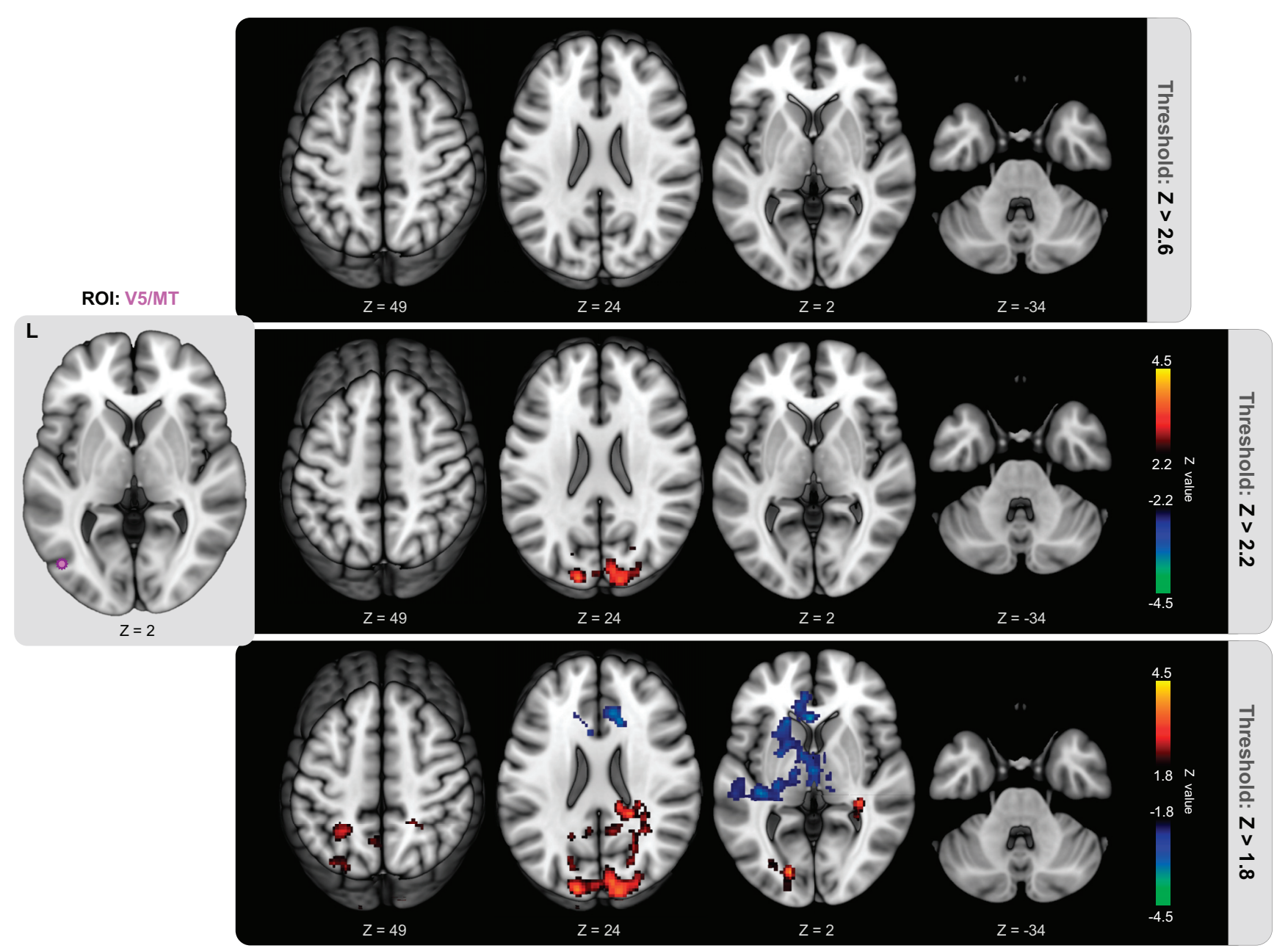

Fig. 7. Changes in FC from day 1 (baseline) to day 2 (after observation) for the control group with respect to the ROI in left V5/MT. No significant clusters appeared when the group-level analysis was thresholded at $Z>2.6$ (top). Clusters appeared only when the threshold was lowered to $Z>2.2$ (middle). Even at lower threshold levels (bottom), the clusters that survive bear no resemblance to those clusters observed in the learning group.

simply due to observing movement error or attending to visual motion but rather are specifically linked to motor learning.

Other controls. To ensure that subjects paid attention to the videos, we instructed them to count the total number of trials in which the tutor performed a reaching movement at the desired speed (indicated by the target disappearing). Reported values were highly accurate for both the learning and control groups, with mean accuracy scores of $95 \%$ and $98 \%$, respectively.

During the motor learning test, the robotic arm applied the FF in a velocity-dependent manner. To ensure that the curvature differences of movements performed in the test FF were due to learning and not to differences in applied force to the hand, we compared movement time and peak tangential velocity (and hence peak force applied by the robot) for each movement block between the groups. No significant differences were observed $(P>0.05)$; thus applied forces to the hand did not differ between the groups during the motor learning test.

The motor learning scores were based on the maximum curvature (PD) of the first three test FF trials relative to (i.e., minus) baseline curvature in the null FF. To assess the robustness of our learning group result, we computed three additional motor learning scores: 1) PD of the first test FF trial relative to baseline curvature, 2) mean PD of the first two test FF trials relative to baseline curvature, and 3) mean PD of the first four test FF trials relative to baseline curvature. We repeated our group-level analysis for the V5/MT seed region, using each of these motor learning scores as the regressor of interest. Each of these analyses yielded clusters very similar to the learning group result presented in Fig. 5.

We further explored the robustness of our learning group result across various parameters used in the neuroimaging preprocessing stage. We ran additional analyses using spatial smoothing kernels of $4 \mathrm{~mm}$ and $5 \mathrm{~mm}$ as well as seed region radii of $4 \mathrm{~mm}$ and $5 \mathrm{~mm}$. Again, these analyses yielded results qualitatively similar to the learning group result presented in Fig. 5.

\section{DISCUSSION}

Here we assessed changes in resting-state FC after subjects observed a tutor learning to reach in a novel FF. We revealed a novel functional network in which changes in FC were correlated with the amount of observational motor learning achieved, as assessed behaviorally after resting-state fMRI scans. We found that left V5/MT showed decreased FC with left cerebellum such that those subjects who learned more through observation exhibited greater decreases in FC. Left 
V5/MT also showed decreased FC with left M1 and left S1 such that those subjects who learned more through observation exhibited greater decreases in FC. Furthermore, in experiment 2 we showed that functional changes in the identified network are not seen in subjects who observed a tutor performing curved movements but not learning. Thus the patterns observed in the identified functional network are not the result of observing movement errors or attending to visual motion, but rather these FC changes are specifically related to motor learning through observation.

FC changes in the identified network may reflect a decoupling between visual and sensory-motor systems, as the visual information about the tutor's movements is transferred to the sensory-motor system for use in motor learning. Subjects who learned more from observation, as assessed by their subsequent motor performance, showed a greater disengagement between V5/MT and cerebellum and between V5/MT and M1 and S1. This visuo-motor decoupling may represent the transference of learning-related activity into motor regions such as cerebellum and sensory-motor cortex for the establishment of internal models of the observed FF (Diedrichsen et al. 2005; Imamizu et al. 2000; Nezafat et al. 2001; Vahdat et al. 2011). Differences in the degree of visual and sensory-motor decoupling may thus reflect differences in the extent to which visual information about the tutor's movements is propagated into the motor domain and mapped onto the observer's own sensorymotor system.

Decreases in FC from day 1 to day 2 were correlated with subsequent behavioral measures of motor learning achieved through observation. While decreases in FC are not as commonly reported in the literature as increases, they are not without precedent (e.g., Vahdat et al. 2014). It is commonplace for resting-state fMRI studies to perform seed-based correlation analyses and report changes in FC after an experimental manipulation. However, if one does not examine the pre- and post-FC values, it cannot be ruled out that reported increases in FC may, in fact, be decreases in FC. As seen here, the subtraction of negative (day 1) FC values from near-zero (day 2) FC values yielded arithmetically positive FC changes. However, upon inspection of the day 1 and day $2 \mathrm{FC}$ values, it is clear that the changes in FC actually correspond to decreases in FC (negative correlations approaching zero). One possible interpretation for this is that on day 1 sensory-motor brain regions and visual motion areas such as V5/MT were coactivated during the baseline null field movements and residual components of this activity were present during the subsequent (day 1) resting-state scan. On day 2, no active movements were completed prior to the resting-state scan; participants had only watched a tutor learning to reach in a FF prior to the day 2 fMRI scan session. Therefore, during the day 2 resting-state scan sensory-motor regions were not primed as strongly as on day 1 , and so perhaps this is why correlations between visual and sensory-motor regions were weaker. However, the observed changes in FC reported here from day 1 to day 2 were reliably related to the degree of learning each participant achieved through observation, and this cannot be explained by differences in sensory-motor priming on day 1 vs. day 2. Furthermore, we did not detect such FC changes in the control group, and this group difference cannot be explained by differences in visual or sensory-motor priming on day 1 vs. day 2 . The findings reported here represent a potential neural basis that specifically links observation of learning with sensorymotor plasticity and associated performance changes in motor learning.

Resting-state fMRI is a powerful technique for studying motor learning. Motor learning does not occur in isolation but rather is accompanied by changes in task performance such as changes in reaction time and decreased attentional demand (Poldrack 2000). This poses a challenge for traditional taskbased fMRI studies of motor learning, which examine activation changes from prelearning to postlearning performance, because they are unable to distinguish those changes in activation that are due to learning from those changes due to differences in performance. Since resting-state fMRI is task free, it is exempt from such performance confounds and observed changes in activation can be directly attributed to learning.

The topographies of resting-state networks closely correspond to those functional networks activated during behavioral tasks (e.g., Fox et al. 2005). Moreover, the coactivation of brain areas during active behavior can modulate subsequent resting-state activity. Task-induced changes in resting-state activity have been demonstrated between visual and frontoparietal attention areas after visual perceptual learning (Lewis et al. 2009), among fronto-parietal and cerebellar networks after visuomotor adaptation (Albert et al. 2009), and among premotor, motor, and cerebellar circuits after FF learning (Vahdat et al. 2011).

There is considerable overlap between the functional network identified in the present study and brain areas involved in motor learning, namely, M1 (Grafton et al. 1992; Steele and Penhune 2010) and cerebellum (Flament et al. 1996; Imamizu et al. 2000; Vahdat et al. 2011). Vahdat and colleagues (2011) have previously examined changes in resting-state FC following FF learning achieved through physical practice using a very similar experimental design (though without a control group). Vahdat and colleagues reported postlearning changes in resting-state FC involving M1, SMA, and cerebellum that were reliably correlated with behavioral scores of active motor learning. Here we observed resting-state FC changes involving visual motion perception area V5/MT, M1, S1, and cerebellum that were related to behavioral scores of motor learning achieved through observation. Thus the present study and that of Vahdat and colleagues both indicate the involvement of M1 and cerebellum in motor learning achieved through either observation or physical practice, respectively. Engagement of these areas during motor learning has been interpreted as reflecting error detection and the execution of corrective movements (e.g., Steele and Penhune 2010). In addition (and in contrast with the study by Vahdat and colleagues), the present study indicates that visual area V5/MT and somatosensory cortex are also engaged when motor learning occurs through observation.

A subset of the brain regions in the identified functional network is also common to the AON, namely, visual area V5/MT, S1, and M1 (Caspers et al. 2010). Moreover, some recent studies also suggest a role for the cerebellum in action observation (e.g., Calvo-Merino et al. 2006; Gazzola and Keysers 2009).

Resting-state networks strongly coincide with underlying structural connectivity. However, some brain regions can exhibit resting-state FC via indirect structural connections 


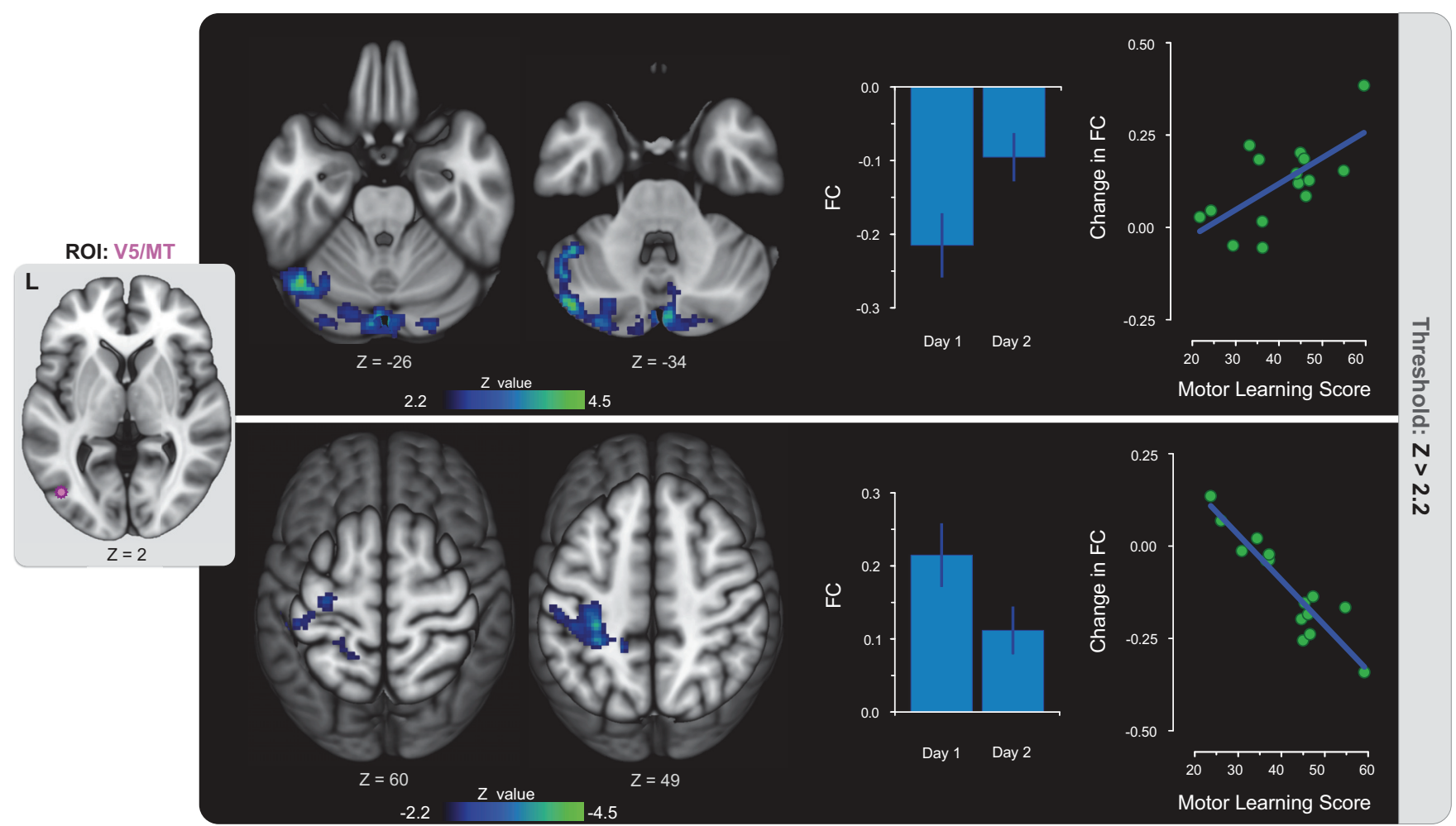

Fig. 8. Changes in FC from day 1 (baseline) to day 2 (after observation) with respect to the ROI in left V5/MT for the learning group. When thresholded at $Z>$ 2.5 , the cluster in left cerebellum extends to right cerebellum (top). When thresholded at $Z>2.2$, the primary sensory-motor cluster extends into left posterior parietal cortex (bottom). Analyses are corrected for familywise error with Gaussian random field theory $(P<0.005)$.

(Vincent et al. 2007). Many of the functional links between V5/MT, cerebellum, M1, and S1 identified in the present study likely arise from such indirect anatomical connections. Neuroanatomical studies have shown that middle temporal area V5/MT projects directly to the cerebellum via the pontine nuclei in the macaque (Ungerleider 1984). V5/MT also projects to the posterior parietal cortex, which in turn relays input to the cerebellum via the pontine nuclei (Glickstein et al. 1980; Langer et al. 1985). The posterior parietal cortex also sends input from V5/MT to S1 and indirectly to M1 (Tanné-Gariépy et al. 2002; Vogt and Pandya 1978). These pathways are also reciprocally connected, with the cerebellum both projecting to and receiving input from the posterior parietal cortex and M1 via the thalamus (Dum and Strick 2003; Thach et al. 1992). Consistent with previous anatomical studies, we observed that the cluster spanning M1 and S1 in the learning group indeed extended into the left posterior parietal cortex (SPL area 5L) when the $Z$ threshold was lowered to 2.2 (Fig. 8).

Neuroimaging studies of action observation and motor learning consistently report ipsilateral or bilateral patterns of cerebellar activity (e.g., Calvo-Merino et al. 2006; Flament et al. 1996; Gazzola and Keysers 2009; Vahdat et al. 2011). In this context, it is perhaps surprising that we observed activation in the contralateral (i.e., left) cerebellar hemisphere in the present study. To further explore the changes in cerebellar activation in our learning group, we tested the sensitivity of the observed pattern of cerebellar FC changes to the chosen statistical threshold. When the $Z$ threshold was lowered to $Z>2.5$, we observed a target cluster in Crus I of the right medial cerebellar hemisphere that showed reliable changes in FC with the V5/MT seed region (Fig. 8).

A functional link between V5/MT and cerebellum has been previously implicated in visual attention to motion. Kellerman and colleagues (2012) showed that effective connectivity between cerebellum (Crus I) and V5/MT was enhanced while subjects attended to moving bars compared with passive fixation. It may be argued that the FC changes between V5/MT and cerebellum observed in the present study may be driven by visual attention to motion. However, the control group showed no FC changes between V5/MT and cerebellum after their observation of the tutor's movement. It is unlikely that visual attention to the tutor's movement, a feature common to both groups, would be the cause of FC changes between V5/MT and cerebellum in the learning group only.

The identified network consisting of V5/MT, cerebellum, M1, and S1 represents a link between visual systems for motion perception and sensory-motor circuits for motor learning. This network may form the basis by which visual information about the movements of others influences sensorymotor circuits for learning to form new motor representations of novel motor skills.

\section{GRANTS}

This research was supported by grants to P. L. Gribble by the Canadian Institutes of Health Research and the Natural Sciences and Engineering Council of Canada.

\section{DISCLOSURES}

No conflicts of interest, financial or otherwise, are declared by the author(s). 


\section{AUTHOR CONTRIBUTIONS}

Author contributions: H.R.M. and P.L.G. conception and design of research; H.R.M. performed experiments; H.R.M. analyzed data; H.R.M. and P.L.G. interpreted results of experiments; H.R.M. prepared figures; H.R.M. drafted manuscript; H.R.M. and P.L.G. edited and revised manuscript; H.R.M. and P.L.G. approved final version of manuscript.

\section{REFERENCES}

Albert NB, Robertson EM, Miall RC. The resting human brain and motor learning. Curr Biol 19: 1023-1027, 2009.

Bernardi NF, Darainy M, Bricolo E, Ostry DJ. Observing motor learning produces somatosensory change. J Neurophysiol 110: 1804-1810, 2013.

Biswal B, Yetkin FZ, Haughton VM, Hyde JS. Functional connectivity in the motor cortex of resting human brain using echo-planar MRI. Magn Reson Med 34: 537-541, 1995.

Brown LE, Wilson ET, Gribble PL. Repetitive transcranial magnetic stimulation to the primary motor cortex interferes with motor learning by observing. J Cogn Neurosci 21: 1013-1022, 2009.

Buccino G, Binkofski F, Fink GR, Fadiga L, Fogassi L, Gallese V, Seitz RJ, Zilles K, Rizzolatti G, Freund HJ. Action observation activates premotor and parietal areas in a somatotopic manner: an fMRI study. Eur J Neurosci 13: 400-404, 2001.

Buccino G, Solodkin A, Small SL. Functions of the mirror neuron system: implications for neurorehabilitation. Cogn Behav Neurol 19: 55-63, 2006.

Buccino G, Vogt S, Ritzl A, Fink GR, Zilles K, Freund HJ, Rizzolatti G. Neural circuits underlying imitation learning of hand actions: an eventrelated fMRI study. Neuron 42: 323-334, 2004.

Calvo-Merino B, Grèzes J, Glaser DE, Passingham RE, Haggard P. Seeing or doing? Influence of visual and motor familiarity in action observation. Curr Biol 16: 1905-1910, 2006.

Caspers S, Zilles K, Laird AR, Eickhoff SB. ALE meta-analysis of action observation and imitation in the human brain. Neuroimage 50: 1148-1167, 2010.

Cothros N, Köhler S, Dickie EW, Mirsattari SM, Gribble PL. Proactive interference as a result of persisting neural representations of previously learned motor skills in primary motor cortex. J Cogn Neurosci 18: 21672176, 2006.

Cross ES, Kraemer DJ, Hamilton AF, Kelley WM, Grafton ST. Sensitivity of the action observation network to physical and observational learning. Cereb Cortex 19: 315-326, 2009.

Damoiseaux JS, Rombouts SA, Barkhof F, Scheltens P, Stam CJ, Smith SM, Beckmann CF. Consistent resting-state networks across healthy subjects. Proc Natl Acad Sci USA 103: 13848-13853, 2006.

Desikan RS, Ségonne F, Fischl B, Quinn BT, Dickerson BC, Blacker D, Buckner RL, Dale AM, Maguire RP, Hyman BT, Albert MS, Killiany RJ. An automated labeling system for subdividing the human cerebral cortex on MRI scans into gyral based regions of interest. Neuroimage 31: 968-980, 2006.

Diedrichsen J, Balsters JH, Flavell J, Cussans E, Ramnani N. A probabilistic MR atlas of the human cerebellum. Neuroimage 46: 39-46, 2009.

Diedrichsen J, Hashambhoy Y, Rane T, Shadmehr R. Neural correlates of reach errors. J Neurosci 25: 9919-9931, 2005.

di Pellegrino G, Fadiga L, Fogassi L, Gallese V, Rizzolatti G. Understanding motor events: a neurophysiological study. Exp Brain Res 91: 176-180, 1992.

Dum RP, Strick PL. An unfolded map of the cerebellar dentate nucleus and its projections to the cerebral cortex. J Neurophysiol 89: 634-639, 2003.

Eickhoff SB, Stephan KE, Mohlberg H, Grefkes C, Fink GR, Amunts K, Zilles K. A new SPM toolbox for combining probabilistic cytoarchitectonic maps and functional imaging data. Neuroimage 25: 1325-1335, 2005.

Flament D, Ellermann JM, Kim SG, Uğurbil K, Ebner TJ. Functional magnetic resonance imaging of cerebellar activation during the learning of a visuomotor dissociation task. Hum Brain Mapp 4: 210-226, 1996.

Flanagan JR, Johansson RS. Action plans used in action observation. Nature 424: 769-771, 2003.

Fox MD, Snyder AZ, Vincent JL, Corbetta M, Van Essen DC, Raichle ME. The human brain is intrinsically organized into dynamic, anticorrelated functional networks. Proc Natl Acad Sci USA 102: 9673-9678, 2005.

Frey SH, Gerry VE. Modulation of neural activity during observational learning of actions and their sequential orders. J Neurosci 26: 13194-13201, 2006

Gallese V, Fadiga L, Fogassi L, Rizzolatti G. Action recognition in the premotor cortex. Brain 119: 593-609, 1996.
Gallese V, Goldman A. Mirror neurons and the simulation theory of mindreading. Trends Cogn Sci 2: 493-501, 1998.

Gallese V, Keysers C, Rizzolatti G. A unifying view of the basis of social cognition. Trends Cogn Sci 8: 396-403, 2004.

Gazzola V, Keysers C. The observation and execution of actions share motor and somatosensory voxels in all tested subjects: single-subject analyses of unsmoothed fMRI data. Cereb Cortex 19: 1239-1255, 2009.

Ghahramani Z, Wolpert DM, Jordan MI. Generalization to local remappings of the visuomotor coordinate transformation. J Neurosci 16: 70857096, 1996.

Glickstein M, Cohen JL, Dixon B, Gibson A, Hollins M, Labossiere E, Robinson F. Corticopontine visual projections in macaque monkeys. $J$ Comp Neurol 190: 209-229, 1980.

Goldstein MH, King AP, West MJ. Social interaction shapes babbling: testing parallels between birdsong and speech. Proc Natl Acad Sci USA 100: 8030-8035, 2003.

Grafton ST, Mazziotta JC, Presty S, Friston KJ, Frackowiak RS, Phelps ME. Functional anatomy of human procedural learning determined with regional cerebral blood flow and PET. J Neurosci 12: 2542-2548, 1992.

Higuchi S, Holle H, Roberts N, Eickhoff SB, Vogt S. Imitation and observational learning of hand actions: prefrontal involvement and connectivity. Neuroimage 59: 1668-1683, 2012.

Iacoboni M, Woods RP, Brass M, Bekkering H, Mazziotta JC, Rizzolatti G. Cortical mechanisms of human imitation. Science 286: 2526-2528, 1999.

Imamizu H, Miyauchi S, Tamada T, Sasaki Y, Takino R, Pütz B, Yoshioka T, Kawato M. Human cerebellar activity reflecting an acquired internal model of a new tool. Nature 403: 192-195, 2000.

Kellermann T, Regenbogen C, De Vos M, Mößnang C, Finkelmeyer A, Habel U. Effective connectivity of the human cerebellum during visual attention. J Neurosci 32: 11453-11460, 2012.

Kuhl PK. Early language acquisition: cracking the speech code. Nat Rev Neurosci 5: 831-843, 2004.

Kuhl PK, Williams KA, Lacerda F, Stevens KN, Lindblom B. Linguistic experience alters phonetic perception in infants by 6 months of age. Science 255: 606-608, 1992.

Langer T, Fuchs AF, Scudder CA, Chubb MC. Afferents to the flocculus of the cerebellum in the rhesus macaque as revealed by retrograde transport of horseradish peroxidase. J Comp Neurol 235: 1-25, 1985.

Lewis CM, Baldassarre A, Committeri G, Romani GL, Corbetta M. Learning sculpts the spontaneous activity of the resting human brain. Proc Natl Acad Sci USA 106: 17558-17563, 2009.

Malfait N, Valyear KF, Culham JC, Anton JL, Brown LE, Gribble PL. fMRI activation during observation of others' reach errors. J Cogn Neurosci 22: 1493-1503, 2010.

Mattar AA, Gribble PL. Motor learning by observing. Neuron 46: 153-160, 2005.

Nezafat R, Shadmehr R, Holcomb HH. Long-term adaptation to dynamics of reaching movements: a PET study. Exp Brain Res 140: 66-76, 2001.

Poldrack RA. Imaging brain plasticity: conceptual and methodological issues-a theoretical review. Neuroimage 12: 1-13, 2000.

Poldrack RA, Mumford JA. Independence in ROI analysis: where is the voodoo? Soc Cogn Affect Neurosci 4: 208-213, 2009.

Prinz W. Perception and action planning. Eur J Cogn Psychol 9: 129-154, 1997.

Prinz W. What re-enactment earns us. Cortex 42: 515-517, 2006.

Rizzolatti G, Fadiga L. Grasping objects and grasping action meanings: the dual role of monkey rostroventral premotor cortex (area f5). Novartis Found Symp 218: 81-95, 1998.

Rizzolatti G, Fadiga L, Gallese V, Fogassi L. Premotor cortex and the recognition of motor actions. Brain Res Cogn Brain Res 3: 131-141, 1996.

Sale P, Franceschini M. Action observation and mirror neuron network: a tool for motor stroke rehabilitation. Eur J Phys Rehabil Med 48: 313-318, 2012.

Scheidt RA, Reinkensmeyer DJ, Conditt MA, Rymer WZ, Mussa-Ivaldi FA. Persistence of motor adaptation during constrained, multi-joint, arm movements. J Neurophysiol 84: 853-862, 2000.

Shadmehr R, Mussa-Ivaldi FA. Adaptive representation of dynamics during learning of a motor task. J Neurosci 14: 3208-3224, 1994.

Steele CJ, Penhune VB. Specific increases within global decreases: a functional magnetic resonance imaging investigation of five days of motor sequence learning. J Neurosci 30: 8332-8341, 2010.

Stefan K, Cohen LG, Duque J, Mazzocchio R, Celnik P, Sawaki L, Ungerleider L, Classen J. Formation of a motor memory by action observation. J Neurosci 25: 9339-9346, 2005. 
Strafella AP, Paus T. Modulation of cortical excitability during action observation: a transcranial magnetic stimulation study. Neuroreport 11: 2289-2292, 2000.

Takahashi CD, Scheidt RA, Reinkensmeyer DJ. Impedance control and internal model formation when reaching in a randomly varying dynamical environment. J Neurophysiol 86: 1047-1051, 2001.

Tanné-Gariépy J, Rouiller EM, Boussaoud D. Parietal inputs to dorsal versus ventral premotor areas in the macaque monkey: evidence for largely segregated visuomotor pathways. Exp Brain Res 145: 91-103, 2002.

Thach WT, Goodkin HP, Keating JG. The cerebellum and the adaptive coordination of movement. Annu Rev Neurosci 15: 403-442, 1992.

Ungerleider LG, Desimone R, Galkin TW, Mishkin M. Subcortical projections of area MT in the macaque. J Comp Neurol 223: 368-386, 1984.

Vahdat S, Darainy M, Milner TE, Ostry DJ. Functionally specific changes in resting-state sensorimotor networks after motor learning. $J$ Neurosci 31: 16907-16915, 2011

Vahdat S, Darainy M, Ostry DJ. Structure of plasticity in human sensory and motor networks due to perceptual learning. J Neurosci 34: 2451-2463, 2014.
Vincent JL, Patel GH, Fox MD, Snyder AZ, Baker JT, Van Essen DC, Zempel JM, Snyder LH, Corbetta M, Raichle ME. Intrinsic functiona architecture in the anaesthetized monkey brain. Nature 447: 83-86, 2007.

Vogt BA, Pandya DN. Cortico-cortical connections of somatic sensory cortex (areas 3, 1 and 2) in the rhesus monkey. J Comp Neurol 177: 179-191, 1978.

Watkins KE, Strafella AP, Paus T. Seeing and hearing speech excites the motor system involved in speech production. Neuropsychologia 41: 989994, 2003.

Watson JD, Myers R, Frackowiak RS, Hajnal JV, Woods RP, Mazziotta JC, Shipp S, Zeki S. Area V5 of the human brain: evidence from a combined study using positron emission tomography and magnetic resonance imaging. Cereb Cortex 3: 79-94, 1993.

Williams A, Gribble PL. Observed effector-independent motor learning by observing. J Neurophysiol 107: 1564-1570, 2012.

Zeki S, Watson JD, Lueck CJ, Friston KJ, Kennard C, Frackowiak RS. A direct demonstration of functional specialization in human visual cortex. $J$ Neurosci 11: 641-649, 1991.

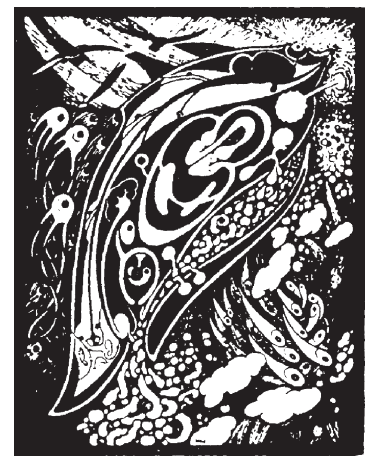

